\title{
Major issues involved in the evaluation of leprosy control programmes through MDT
}

\author{
C K RAO
}

\section{Introduction}

In the past 4-5 years countries where leprosy is endemic have increasingly adopted multidrug therapy (MDT) in the treatment of leprosy. The broad aim of MDT is to interrupt the transmission of infection through early case detection and regular and complete treatment, and also to prevent disabilities and deformities. Reports on effectiveness, safety and patient acceptability from countries implementing MDT have continued to be positive, and also show that MDT has increased community confidence in the curability of the disease, which promotes voluntary self-reporting of patients.

Planning and evaluation are managerial tools that contribute to the success of leprosy control programmes. Evaluation helps further prospective planning to be effective by identifying achievements or shortcomings and highlighting the points that could improve programme performance.

The implementation of MDT for leprosy cases demands a highly sensitive monitoring and evaluation system to ensure the programme's smooth and coordinated progress. The use of expensive and effective drugs under supervision for relatively long periods makes treatment monitoring a crucial component of the programme. The correct time of programme inputs, including drugs and educational material, is ensured through programme monitoring. Monitoring also helps in reshuffling priorities, dropping unproductive efforts and in indicating neglected areas.

In this paper evaluation has been taken to include both monitoring (day-to-day follow-up of activities) and evaluation. Monitoring is also referred to by some programme designers as internal evaluation and is often in-built in leprosy control programmes. Unlike monitoring, external evaluation is periodic and independent of the programme personnel, which ensures lesser individual bias and greater reliability of data reported.

My experience in planning and participating in the independent evaluation of leprosy programmes in India and Myanmar in recent years is the basis for delineating important issues involved in the evaluation exercise.

\section{Issues}

OBJECTIVES OF EVALUATION

The objectives of the programme may vary from country to country, depending upon the 
aims, strategies, inf rastructure, and duration of the MDT operations, and may include some or all of the following:

-Assessment of case detection progress, case treatment, drug delivery and reasons for patient default.

- Validation of reported data through examination of records and field visits.

-Assessment of the ongoing information system in terms of its promptness and completeness.

-Ascertainment of the technical competence and devotion of the staff involved.

-Determination of the impact of health education, if any, in dispelling ignorance/ prejudices in the community, in promoting regularity of treatment and in retrieving the defaulters.

- Examination of the impact of the measures on the disease.

\section{KEY COMPONENTS IN MDT DELIVERY}

\section{Treatment delivery}

MDT is delivered once a month by the health personnel at predetermined points near the patient's home or in health centres/dispensaries/out-patient departments of hospitals, either to all eligible patients or only to selected patients, as decided in a country's programme.

\section{Case detection}

This is achieved through the promotion of voluntary self-reporting of patients, through active surveys, or by both methods, envisaged under the programme chosen.

\section{Patient card maintenance}

This should indicate the clinical/bacteriological status before, during and after MDT.

\section{Case treatment}

It should be specified which MDT regimes are followed for multibacillary (MB) and paucibacillary (PB) cases, and the regularity of MDT and monitoring drug intake by patients under the programme.

\section{Record maintenance}

Data recording and reporting systems should be maintained at different levels and there should be officers responsible for this, and feedback should be given to the senior officers clarifying the strong and weak points in the reports.

\section{Health education}

This occupies a high priority in the success of MDT-though it is the responsibility of every health worker involved in the programme to educate the patients, their families and 
the community, this may receive very little attention from most of them, especially in integrated programmes. Voluntary self-reporting of cases, a high compliance rate and a high rate of treatment completion reflect how effective the education component is in the community, considering patient awareness, patient participation and community acceptance.

\section{Leprosy profile}

General information on leprosy prevalence and other epidemiological indices in the area before and during MDT should be available.

\section{Infrastructure}

Enumerating general health services personnel and/or special leprosy workers involved/ available for a leprosy programme and designating their job training status.

\section{Voluntary organizations}

These have to be active participants in monitoring and evaluation from the programme planning stage when working for leprosy control in a country

\section{Monitoring methodology}

The existing information recording and reporting procedures followed to monitor a leprosy control programme may vary from country to country. Most programmes that originated as strong vertical programmes continue to have a comprehensive reporting system compatible with/adopted from OMSLEP. Appropriately most integrated programmes have a simple and practical reporting system that include the core activities of case detection and case treatment as a part of health care reporting. The aim of leprosy information systems is to give timely though roughly correct figures rather than unduly precise but delayed data.

\section{Sources of information}

These should be leprosy patient cards and registers, leprosy survey data, surveillance information and supervisors' reports.

\section{Training of monitoring personnel}

All health workers responsible for monitoring data collection and for supervision should obtain their skills during job training for leprosy control.

\section{Selection of indicators for monitoring leprosy programmes}

These may vary from country to country based upon the programme aims, strategies and the infrastructure implementing it. The broad goals of MDT in leprosy control programmes should be to provide in full the course of MDT to leprosy cases, therefore 
certain minimum indicators must be monitored. The following five indicators suggested to be 'required' at the WHO Consultation on Technical and Operational aspects of leprosy in Male, Maldives, in June 1990, are considered appropriate as minimum indicators: (i) prevalence; (ii) case detection; (iii) the proportion of patients with disability grade II among newly detected cases; (iv) MDT coverage, i.e. the proportion on MDT against all registered cases for chemotherapy; and (v) MDT completion, i.e. the proportion who have completed MDT among those put on MDT. Advanced programmes could develop additional indicators that were suggested in the report of the WHO Study Group on the Epidemiology of Leprosy in Relation to Control (TRS716). Operational criteria for definition of a n active case for computing prevalence suggested by the Sixth Expert Committee on Leprosy (TRS768) would be appropriate to ensure uniformity, to define the targets for MDT and to determine the disease trends following MDT programmes.

\section{Supervision}

This is central to monitoring. Supervision ranges from validation of disease diagnosis, classification, activity, treatment delivery, treatment intake, detection and the management of reactions, skin-smear results and also logistics-delivery of drugs and transport. Part-time or full-time supervisors at different levels are identified and trained in the supervisory skills and techniques of leprosy control programmes.

\section{Feedback on reports}

Regular feedback from supervisors on their observations concerning both strong and weak points of the programme and comments on reports to lower reporting echelons, though not involved in decision-making, improves the programme performance.

\section{STRENGTHENING MONITORING SYSTEM}

An in-built monitoring system is often subjected to decay, and becomes less effective with time. However, the decay could be minimized and delayed by the periodic training of workers in skills to review critically the data and initiate corrective actions, maintenance of patient cards, encourage effective supervision, a periodic programme review of different levels by the highest administrative authority, listing priority indicators for monitoring, issuing a periodic news letter, maintenance and storage of records, etc.

The Indian programme appears to be unique in having a system of internal evaluation of leprosy programmes by creating regular assessment teams supported by the central programme at the state level and hiring full-time/part-time consultants supported by the WHO at national level. Though a formal review of their contributions has not been done, it is considered that they help to improve the quality of reported data, as well as tackling the operational/administrative problems in time. The programme is considering ways and means of keeping internal evaluators on a continuous basis.

\section{EXTERNAL EVALUATION}

The existence of a leprosy information system is basic to evaluation and monitoring. As 
mentioned earlier, an evaluation undertaken by an expert who is independent of the programme planning and implementation, ensures lesser individual bias and a greater reliability of data.

\section{Objectives}

The objectives of evaluation listed above are perhaps relevant for external evaluation as well as with varying priorities. Broad objectives of evaluation are two-fold; one is determination of operational efficiency, i.e. to examine if what was planned or expected was in fact carried out, and the other is the determination of the impact of the control measures on the selected indices, i.e. whether what was expected in terms of selected indices did actually happen.

\section{Collateral benefits}

An element of healthy competition among the staff, especially middle-level managers, raises the morale of the peripheral staff, motivating health administrators, health planners and politicians for their increased support. Through their active participation it educates the administrative medical officers at state/division level on the strengths and weaknesses of the programme in their area vis-à-vis at the national level.

\section{Sources of data}

Records and reports maintained at all (peripheral to national) levels on the programme activities to delineate the leprosy profile.

Annual reports of the programme for the last 2-3 years.

Monthly/quarterly reports, if any, for the current year and previous year.

Leprosy patient data/cards maintained at villages/health centres.

Data obtained from discussions with programme managers-national, provincial, divisional, township/regency/district levels.

Interviews with health workers, supervisors, leprosy patients and community members.

\section{Questionnaires for data collection}

Appropriate questionnaires are constructed and pretested by the country programme manager taking account of the terms of ref erence for independent evaluation and the time available for evaluation. The questionnaires are used for interviewing programme managers, medical officers, supervisors and health workers involved in leprosy control at different levels to determine their competence and contribution. Questionnaires are also used for interrogation of leprosy patients and community members to determine the level of their awareness, participation in the programme activities, perception on social aspects of leprosy, etc. Questionnaires are also developed to collect appropriate data on leprosy control programmes at different levels. About 14 or 15 questionnaires were used in the three Indian programme evaluations and 7 in the Myanmar programme evaluation. Questionnaires to ascertain the leprosy profile from the states in India and divisions in Myanmar were sent to all concerned with the central programme 2 weeks before the 
proposed field visits with a request to place the data at the disposal of the evaluators, should they visit that particular state/division. This ensured a timely submission of data and self-review of the data in the states/divisions irrespective of the actual visit of evaluators.

\section{Sample selection}

Effective evaluation, unlike monitoring, can only be carried out in a small sample because of time and funding constraints. Samples for field visits were selected by a WHO consultant independent of the programme managers both in India and Myanmar. Random samples of states and then districts were selected in India to ensure wide coverage of the country after stratification by levels of leprosy endemicity, varying organizational inf rastructure and duration of MDT activities in force. A total of 10 multibacillary $(\mathrm{MB})$ and 10 paucibacillary $(\mathrm{PB})$ patients and 20 community members in 2 villages selected by the evaluators were interrogated in each of the districts assigned for evaluation in India.

In Myanmar $5 \mathrm{MB}$ and $5 \mathrm{~PB}$ patients were chosen to be interviewed in 2 villages in each township selected for evaluation. Voluntary organizations involved in leprosy control efforts in the districts selected for evaluation were also included for evaluation of the Indian programme. No voluntary organization was involved in leprosy control work in the townships selected for evaluation in Myanmar.

\section{Selection of evaluators}

In India teams of 3 experts each have helped to improve competent evaluation of programme management (by a health administrator), impact assessment (by an epidemiologist), and validation of reported data (by a leprologist) besides giving other administrative and operational advantages of a team approach. In India 9-12 teams were formed for the three evaluations of the programme in 1986, 1987 and 1989. As mentioned above, each team had the services of a leprologist/leprosy control expert provided by the WHO from outside India. National evaluators were drawn from directors of health services of states or the equivalent, professors of community health or the equivalent and similar experts working with voluntary organizations whose involvement motivated them to support the programme.

In Myanmar, regional leprosy officers from outside their divisions were involved as evaluators along with a WHO consultant, the latter having selected the divisions and townships on a random sampling basis, and 2 teams of 3 experts were formed.

Should in-depth evaluation of some areas or some component of the programme be considered necessary, suitable experts as evaluators have to be recruited.

It is necessary to ensure that the evaluators are adequately briefed at the start of the evaluation so that they are able to fill the questionnaires and collect the requisite data correctly and uniformly. Briefing was given for 2 days both in the Indian and the Myanmar evaluations.

\section{Duration of evaluation}

It is convenient to complete the evaluation — travel, briefing and report-in 10-15 days. 
With appropriate planning and preparation this period was found to be satisfactory. Disruption of routine programme activities are marginal in brief evaluations. When a smaller number of evaluators/teams are available, evaluation has to be prolonged over a relatively longer period. The Indian and Myanmar programme evaluations were all completed within 15 days. Funds for these evaluations were available from their respective WHO country budgets.

\section{Collection of data}

Appropriate data are collected by the evaluators from the reports using the assigned terms of reference. Information on the infrastructure availability against the sanctioned strength, training status of personnel, leprosy prevalence, case detection, case treatment, MDT coverage, MDT completion, health educational activities and their impact, quality of laboratory services, quality of supervision, supply of drugs, mobility, etc. are collected by the evaluators on a sample basis. Evaluators also validate a small sample of reported data on diagnosis, classification, treatment schedules, regularity of drug delivery and drug intake, disease activity, skin-smear results, etc. and record the data on the appropriate questionnaires.

\section{Analysis and interpretation of data}

Evaluators are expected to present orally their findings to the senior health officer of the state/division at the end of the visit. Hence data collected will have to be analysed before leaving the assigned states/divisions. It is a good strategy first to highlight the strengths of the programme, if any, before indicating the areas that need strengthening by the state/ division health administration. On return to the central programme headquarters to report and deliver the duly completed questionnaires the points of view of the state/ division, if any, have to be considered to see if it is necessary to relay them to national level. During oral presentation and debriefing the strengths found are to be projected while also suggesting areas that require urgent corrective action at all levels of the programme, including the central level.

\section{Report and recommendations}

Each evaluator/team has to give a narrative report with the completed questionnaires using the terms of reference assigned for evaluation. Brief, lucid and timely reports including positive features of the programme are helpful to improve/strengthen the programme performance. The evaluation reports have provided valuable support to the Indian programme-strengthening laboratory services; making possible the rapid extension of MDT to a large number of districts; the timely release of funds for health educational activities; giving priority to the filling of vacant posts; the training of personnel; and increasing the budget.

\section{Actions taken on the report}

The major aim of leprosy programme evaluation is to improve its performance. Evaluation guides in decision making. This purpose will not be achieved if the report is 
$60 \mathrm{~s}$ C K Rao

unduly delayed or timely corrective actions are not initiated. Hence it is necessary to review the actions taken on the recommendations of earlier evaluation, if undertaken.

\section{Conclusion}

It may be stated that leprosy evaluation procedures followed in one country could be adopted in another country with only minor modifications, where warranted, to suit the local conditions. 